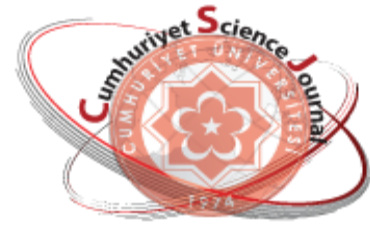

e-ISSN: $2587-246 X$

ISSN: $2587-2680$

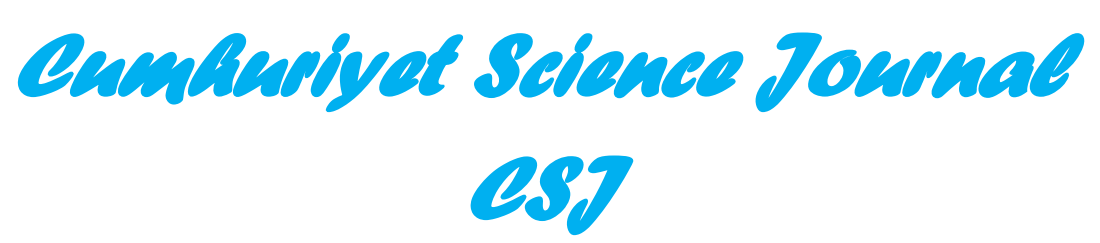

Cumhuriyet Sci. J., Vol.40-4 (2019) 792-801

\title{
3-Zero-Divisor Hypergraph with Respect to an Element in Multiplicative Lattice
}

\author{
Gülşen ULUCAK ${ }^{1}$ \\ ${ }^{1}$ Department of Mathematics, Gebze Technical University, P.K 41400, Gebze-Kocaeli, TURKEY
}

Received: 19.11.2018; Accepted: 23.10.2019

http://dx.doi.org/10.17776/csj.485085

\begin{abstract}
Let $L$ be a multiplicative lattice and $z$ be a proper element of $L$. We introduce the 3-zero-divisor hypergraph of $L$ with respect to $z$ which is a hypergraph whose vertices are elements of the set $\left\{\begin{array}{c}x_{1} \in L-\{z\} \\ \begin{array}{c}x_{1} x_{2} x_{3} \leq z \Rightarrow x_{1} x_{2} \ddagger z, x_{2} x_{3} \$ z \text { and } x_{1} x_{3} \ddagger z \\ \text { for some } x_{2}, x_{3} \in L-\{z\}\end{array}\end{array}\right\}$ where distinct vertices $x_{1}, x_{2}$ and $x_{3}$ are adjacent, that is, $\left\{x_{1}, x_{2}, x_{3}\right\}$ is a hyperedge if and only if $x_{1} x_{2} x_{3} \leq z \Rightarrow x_{1} x_{2} \$ z, x_{2} x_{3} \$ z$ and $x_{1} x_{3} \$ z$. Throughout this paper, the hypergraph is denoted by $H_{3}(L, z)$. We investigate many properties of the hypergraph over a multiplicative lattice. Moreover, we find a lower bound of diameter of $H_{3}(L, z)$ and obtain that $H_{3}(L, z)$ is connected.
\end{abstract}

Keywords: 3-Zero-Divisor Hypergraph, Complete n-partite Hypergraph.

\section{Çarpımsal Kafeslerde Bir Eleman ile İlgili 3-lü Sıfır Bölen Hipergrafı}

Özet. $L$ bir çarpımsal kafes ve $z, L$ nin bir has elemanı olsun. $z$ ile ilgili $L$ nin 3-lü sıfır bölen hipergrafını tanıttık öyle ki bu hipergrafin köşeleri $\left\{x_{1} \in L-\{z\} \mid \begin{array}{c}x_{1} x_{2} x_{3} \leq z \Rightarrow x_{1} x_{2} \$ z, x_{2} x_{3} \$ z \text { ve } x_{1} x_{3} \$ z \\ \text { herhangi } x_{2}, x_{3} \in L-\{z\} \text { için }\end{array}\right\}$ kümesinin elemanlarıdır ki burada $x_{1}, x_{2}$ ve $x_{3}$ komşudur, yani, $\left\{x_{1}, x_{2}, x_{3}\right\}$ bu hipergarfın bir hiperkenarıdır ancak ve ancak $x_{1} x_{2} x_{3} \leq z \Rightarrow x_{1} x_{2} \$ z, x_{2} x_{3} \$ z$ ve $x_{1} x_{3} \$ z$. Bu çalışma boyunca, bu hipergrafi $H_{3}(L, z)$ ile göstereceğiz. Çarpımsal bir kafes üzerinde bu hipergrafın birçok özelliğini araştırdık. Ayrıca, $H_{3}(L, z)$ nin diametresinin bir alt sınırını bulduk ve bu hipergrafin bağlantılı olduğunu gösterdik.

Anahtar Kelimeler: 3-lü Sıfır Bölen Hipergraf, n-parçalı Tam Hipergraf.

\section{INTRODUCTION}

A complete lattice $L$ is called multiplicative lattice if there exists a commutative, associative, completely join distributive product on the lattice with the compact greatest element $1_{L}$, which is the multiplicative identity, and the least element $0_{L}$. It can be easily seen that $L / a=\{b \in L \mid a \leq$ $b$ \} is a multiplicative lattice with the product $x \circ y=x y \vee a$ where $L$ is multiplicative lattice and $a \in L$. Note that $0_{L / z}=z$. D.D. Anderson and the current authors have studied on multiplicative lattices in a series of articles [1-4]. An element $a \in L$ is said to be proper if $a<1_{L}$. A proper element $p \in L$ is called a prime element if $a b \leq p$ implies $a \leq p$ or $b \leq p$, where $a, b \in L$. Then $p$ is called 2-absorbing element of $L$ if $x_{1} x_{2} x_{3} \leq p$ for some $x_{1}, x_{2}$ and $x_{3}$ in L, then $x_{1} x_{2} \leq p$ or $x_{1} x_{3} \leq p$ or $x_{2} x_{3} \leq p$.

Let a finite set $V$ be a vertex set and $E(V)=\{(u, v) \mid u, v \in V, u \neq v\}$. A pairwise $G=(V, E)$ is called a graph on $V$ where $E \subseteq E(V)$. The elements of $V$ are the vertices of $G$, and those of $E$ the 
edges of $G$. Consider that the edges $(x, y)$ and $(y, x)$ denote the same edge (For more information, see [3-8].

A hypergraph $H$ is a pair $(V, E)$ of disjoint sets, where the elements of $E$ are nonempty subsets of $V$. The elements of $V$ are called the vertices of $H$ and the elements of $E$ are called the hyperedges of $H$. If the size of any hyperedge $e$ in the hypergraph $H$ is $n$, then $H$ is called $n$-uniform hypergraph. Let $H$ be an $n$-uniform hypergraph. An alternating sequence of distinct vertices and hyperedges is called a path with the form $v_{1}, e_{1}, v_{2}, e_{2}, \ldots, v_{m}$ such that $v_{i}, v_{i+1}$ are in $e_{i}$ for all $1 \leq i \leq m-1$. The length of a path is the number of hyperedges of it. The distance $d(x, y)$ between two vertices $x$ and $y$ of $H$ is the length of the shortest path from $x$ to $y$. If no such path between $x$ and $y$ exists, then $d(x, y)=\infty$. The diameter $\operatorname{diam}(H)$ of $H$ is the greatest distance between any two vertices. The hypergraph $H$ is said to be connected if $\operatorname{diam}(H)<\infty$. A cycle in a hypergraph $H$ is an alternating sequence of distinct vertices and hyperedges of the form $v_{1}, e_{1}, v_{2}, e_{2}, \ldots, v_{m}, e_{m}, v_{1}$ such that $v_{i}, v_{i+1} \in e_{i}$ and $v_{m}, v_{1} \in e_{m}$ for all $1 \leq i \leq m$. The girth $\operatorname{gr}(H)$ of a hypergraph $H$ containing a cycle is the smallest size of the length of cycles of $H$. (For more information, see [5]). A hypergraph $H$ is called trivial if it has a single vertex and also it is called empty if it has no hyperedges.

The concept of a zero-divisor graph of a commutative ring was first introduced in [6]. Let $R$ be a commutative ring and $k \geq 2$ be an integer. A nonzero nonunit element $x_{1}$ in $R$ is said to be a $k$-zerodivisor in $R$ if there are $k-1$ distinct nonunit elements $x_{2}, x_{3}, \ldots, x_{k}$ in $R$ different from $x_{1}$ such that $x_{1} x_{2} x_{3} \ldots x_{k}=0$ and the product of no elements of any proper subset of $A=\left\{x_{1}, x_{2}, x_{3}, \ldots, x_{k}\right\}$ is zero. The set of $k$-zero divisor elements of $R$ is denoted by $Z_{k}(R)$. Let $I$ be a proper ideal of $R$. The 3-zero-divisor hypergraph of $R$ with respect to $I$, denoted by $H_{3}(R, I)$, is the hypergraph whose vertices are the set $\left\{x_{1} \in R \backslash I \mid x_{1} x_{2} x_{3} \in I\right.$ for some $x_{2}, x_{3} \in R \backslash I$ such that $x_{1} x_{2} \notin I, x_{2} x_{3} \notin$ $I$ and $\left.x_{1} x_{3} \notin I\right\}$ where distinct vertices $x_{1}, x_{2}$ and $x_{3}$ are adjacent if and only if $x_{1} x_{2} x_{3} \in I, x_{1} x_{2} \notin$ $I, x_{2} x_{3} \notin I$ and $x_{1} x_{3} \notin I$ (See [9]). Let $I$ be a proper ideal of $R$. Recall that $I$ is called a 2-absorbing ideal of $R$ if $x_{1} x_{2} x_{3} \in I$ for some $x_{1}, x_{2}$ and $x_{3}$ in $R$, then $x_{1} x_{2} \in I$ or $x_{2} x_{3} \in I$ or $x_{1} x_{3} \in I$ (For more information, see [10]). Hence $H_{3}(R, I)$ is not empty if and only if $I$ is not a 2-absorbing ideal of $R$ (see Proposition 1 in [9]).

Let $z$ be a proper element of $L$. A proper element $a_{1}$ of $L$ is called $n$-zero divisor element with respect to $z$ in $L$ if there are $n-1$ distinct elements $a_{2}, a_{3}, \ldots, a_{n}$ in $L$ different from $a_{1}$ such that $a_{2} a_{3} \ldots a_{n} \leq z$ and the product of no elements of any proper subset of $A=\left\{a_{1}, a_{2}, \ldots, a_{n}\right\}$ is less than or equals to $z$. The set of all $n$-zero divisor element with respect to $z$ in $L$ is denoted by $Z_{n}(L, z)$. For example, consider the lattice of ideals of $\mathbb{Z}, L=\mathrm{I}(\mathbb{Z})$ the set of all ideals of $\mathbb{Z}$. The ideal (2) is a 3-zero-divisor with respect to (8) in $L$ since (2)(3)(6) $\subseteq(8)$, and the product of no elements of any proper subset of $\{(2),(3),(6)\}$ is contained by (8).

Throughout this paper, we assume that a lattice $L$ is a multiplicative lattice. Let $z$ be a proper element of $L$. The 3-zero-divisor hyper-graph of $L$ with respect to $z$, denoted by $H_{3}(L, z)$, is a hypergraph whose vertices are elements of the set $\left\{\begin{array}{c}x_{1} \in L-\{z\} \\ \begin{array}{c}x_{1} x_{2} x_{3} \leq z \Rightarrow x_{1} x_{2} \$ z, x_{2} x_{3} \$ z \text { and } x_{1} x_{3} \$ z \\ \text { for some } x_{2}, x_{3} \in L-\{z\}\end{array}\end{array}\right\}$ such that distinct vertices $x_{1}, x_{2}$ and $x_{3}$ are adjacent, that is, $\left\{x_{1}, x_{2}, x_{3}\right\}$ is a hyperedge if and only if $x_{1} x_{2} x_{3} \leq z \Rightarrow x_{1} x_{2} \$ z$, $x_{2} x_{3} \$ z$ and $x_{1} x_{3} \npreceq z$. It can be seen that $H_{3}(L, z)$ is a 3-uniform hypergraph. In this paper, we show that $H_{3}(L, z)$ is empty if and only if $z$ is a 2-absorbing element of $L$ and also, $H_{3}(L / z)$ is empty 
hypergraph if and only if $H_{3}(L, z)$ is empty hypergraph. Then we give that $H_{3}(L, Z)$ is connected and $\operatorname{diam}\left(H_{3}(L, z)\right) \leq 4$. Additionally, we show that $H_{3}(L, z)$ is a complete 3-partite hypergraph if $p_{1}, p_{2}$ and $p_{3}$ are prime elements of $L$ and $z=p_{1} \wedge p_{2} \wedge p_{3} \neq 0_{L}$ and the converse is true if $L$ is reduced lattice. Finally, we see that $H_{3}(L, z)$ has no cut-point.

\section{ZERO DIVISOR HYPERGRAPH H_3 (L,z) WITH RESPECT TO z}

Definition 1. Let $z$ be a proper element of $L$. The 3-zero-divisor hypergraph of $L$ with respect to $z$ is a hypergraph whose vertices are elements of the set $\left\{x_{1} \in L-\{z\} \mid \begin{array}{c}x_{1} x_{2} x_{3} \leq z \Rightarrow x_{1} x_{2} \ddagger z, x_{2} x_{3} \$ z \text { and } x_{1} x_{3} \$ z \\ \text { for some } x_{2}, x_{3} \in L-\{z\}\end{array}\right\}$. Also, distinct vertices $x_{1}, x_{2}$ and $x_{3}$ are adjacent, that is, $\left\{x_{1}, x_{2}, x_{3}\right\}$ is a hyperedge if and only if $x_{1} x_{2} x_{3} \leq z \Rightarrow x_{1} x_{2} \ddagger z, x_{2} x_{3} \ddagger$ $z$ and $x_{1} x_{3} \$ z$. Throughout this paper, the hypergraph is denoted by $H_{3}(L, z)$.

Let $z=0_{L}$. Then it is clear that $H_{3}(L)=H_{3}\left(L, 0_{L}\right)$ is the hypergraph whose vertices are elements of the $\operatorname{set}\left\{x_{1} \in Z_{3}(L) \mid \begin{array}{c}x_{1} x_{2} x_{3}=0_{L} \Rightarrow x_{1} x_{2} \neq 0_{L}, x_{2} x_{3} \neq 0_{L} \text { and } x_{1} x_{3} \neq 0_{L} \\ \text { for some } x_{2}, x_{3} \in Z_{3}(L)\end{array}\right\}$ where distinct vertices $x_{1}, x_{2}$ and $x_{3}$ are adjacent if and only if $x_{1} x_{2} x_{3}=0_{L} \Rightarrow x_{1} x_{2} \neq 0_{L}, x_{2} x_{3} \neq 0_{L}$ and $x_{1} x_{3} \neq$ $0_{L}$

The hypergraphs $H_{3}(R)$ in [5] and $H_{3}(R, I)$ in [10], which are defined on a commutative ring $R$ and a proper ideal $I$ of $R$, are examples for the hypergraph $H_{3}(L, z)$.

We obtain the following results with the above definition and the definition of 2-absorbing element in $L$.

Proposition 1. Let $z$ be a proper element of $L$. Then the following statements hold:

1) $H_{3}(L, z)$ is empty hypergraph if and only if $z$ is a 2-absorbing element of $L$.

2) $H_{3}(L / z)$ is empty hypergraph if and only if $H_{3}(L, z)$ is empty hypergraph.

Proof. 1). $(\Rightarrow)$ : Let $H_{3}(L, z)$ be empty hypergraph. Suppose that $z$ is not a 2-absorbing element of $L$. Take $x_{1} x_{2} x_{3} \leq z$ for some $x_{1}, x_{2}, x_{3} \in L$. Then we get $x_{1} x_{2} \$ z, x_{2} x_{3} \$ z$ and $x_{1} x_{3} \$ z$. Hence $e=\left\{x_{1}, x_{2}, x_{3}\right\}$ is a hyperedge of $H_{3}(L, z)$, a contradiction.

$(\Leftarrow)$ : It is obvious.

2). $(\Rightarrow)$ : Assume that $H_{3}(L, z)$ is not an empty hypergraph. Then it has a hyperedge $e=\left\{x_{1}, x_{2}, x_{3}\right\}$. Consider $x_{1} \vee z, x_{2} \vee z, x_{3} \vee z \in L / z$. It is clear that $x_{1} \vee z, x_{2} \vee z, x_{3} \vee z$ are different from $z$. Then we have that $\left(x_{1} \vee z\right)\left(x_{2} \vee z\right)\left(x_{3} \vee z\right)=0_{L / z},\left(x_{1} \vee z\right)\left(x_{2} \vee z\right) \neq 0_{L / z},\left(x_{2} \vee z\right)\left(x_{3} \vee z\right) \neq 0_{L / z}$ and $\left(x_{1} \vee z\right)\left(x_{3} \vee z\right) \neq 0_{L / z}$. Thus $e^{\prime}=\left\{x_{1} \vee z, x_{2} \vee z, x_{3} \vee z\right\}$ is a hyperedge of $H_{3}(L / z)$, a contradiction.

$(\Leftarrow)$ : Let $H_{3}(L / z)$ be not an empty hypergraph. Then it has a hyperedge $e=\left\{y_{1}, y_{2}, y_{3}\right\}$ for some $y_{1}, y_{2}, y_{3} \in V\left(H_{3}(L / z)\right)$. Then $y_{1} \circ y_{2} \circ y_{3}=0_{L / z}$, that is, $y_{1} y_{2} y_{3} \leq z$ and since $y_{1} \circ y_{2}, y_{2} \circ y_{3}$ and $y_{1} \circ y_{3}$ are different from $0_{L / z}$, then $y_{1} y_{2}, y_{2} y_{3}, y_{1} y_{3} \nsubseteq z$. Therefore, $e=\left\{y_{1}, y_{2}, y_{3}\right\}$ is a hyperedge of $H_{3}(L, z)$, a contradiction. 
Theorem 1. Let $H_{3}(L, z)$ be a 3-zero-divisor hypergraph of $L$ with respect to $z$. If $x^{2} \ddagger z$ for each 3-zero-divisor $x \in L$ with respect to $z$, then $H_{3}(L, z)$ is connected and $\operatorname{diam}\left(H_{3}(L, z)\right) \leq 4$. Furthermore, if $H_{3}(L, z)$ has a cycle, then $\operatorname{gr}\left(H_{3}(L, z)\right) \leq 9$.

Proof. Let $e_{1}=\left\{x_{1}, x_{2}, x_{3}\right\}$ and $e_{2}=\left\{y_{1}, y_{2}, y_{3}\right\}$ be hyperedges of $H_{3}(L, z)$. If $e_{1} \cap e_{2} \neq \emptyset$, the proof is completed. Assume that $e_{1} \cap e_{2}=\emptyset$. We show that there are hyperedges $e_{3}, e_{4}$ such that they satisfy one of the followings:

$$
\begin{aligned}
& \boldsymbol{e}_{3} \cap \boldsymbol{e}_{1} \neq \emptyset, \boldsymbol{e}_{3} \cap \boldsymbol{e}_{2} \neq \varnothing \\
& e_{3} \cap e_{1} \neq \emptyset, e_{4} \cap e_{2} \neq \varnothing, e_{4} \cap e_{3} \neq \emptyset
\end{aligned}
$$

Assume that $G$ is the partite graph such that $V(G)=e_{1} \cup e_{2}$ and $x_{i} y_{j} \in E(G)$ if and only if $x_{i} y_{j} \leq$ $z$.

Assume that $G$ has two isolated vertices such that one is in $e_{1}$ and the other is in $e_{2}$. Let $\operatorname{deg}_{G}\left(x_{3}\right)=$ $\operatorname{deg}_{G}\left(y_{3}\right)=0$. Suppose that there is $a \in\left\{x_{1}, x_{2}, y_{1}, y_{2}\right\}$ where $x_{3} y_{3} a \leq z$. Then $e_{3}=\left\{x_{3}, y_{3}, a\right\}$ is a hyperedge which holds the condition (1). Let the case not satisfy. If $x_{3} y_{3} \notin\left\{x_{1}, x_{2}, y_{1}, y_{2}\right\}$, then $e_{3}=\left\{x_{1}, x_{2}, x_{3} y_{3}\right\}$ and $e_{4}=\left\{y_{1}, y_{2}, x_{3} y_{3}\right\}$ are two hyperedges which satisfy the condition (2). In the contrary case, without loss of generality (wlog.), suppose that $x_{3} y_{3}=x_{1}$. Hence $e_{3}=$ $\left\{x_{1}, y_{1}, y_{2}\right\}$ is a hyperedge satisfying the condition (1). Consequently, $H_{3}(L, z)$ is connected. Now, we show that $\operatorname{diam}\left(H_{3}(L, z)\right) \leq 4$. We consider the number of edges $G$ for the rest of the proof.

Case 1. Assume that $|E(G)| \leq 2$. Then $G$ has two isolated vertices such that one is in $e_{1}$ and the other is in $e_{2}$.

Case 2. Let $|E(G)|=3$. Take account of the next four different subcases for this case:

Case 2.1: $\operatorname{Let}_{\operatorname{deg}}(a)=1$ for each vertex $a$ of $G$. Assume that $E(G)=\left\{x_{1} y_{1}, x_{2} y_{2}, x_{3} y_{3}\right\}$. We consider $\left\{x_{1}, x_{2} y_{3}, y_{1} \vee y_{2}\right\}$. If $x_{1}=x_{2} y_{3}$, then $x_{1} y_{2}=x_{2} y_{3} y_{2} \leq z$, a contradiction. If $x_{1}=y_{1} \vee$ $y_{2}$, then $y_{1} x_{2} x_{3} \leq z$. Thus $e_{3}=\left\{y_{1}, x_{2}, x_{3}\right\}$ satisfies the condition (1). If $y_{1} \vee y_{2}=x_{2} y_{3}$, then $x_{1} y_{2} x_{3} \leq z$ and so the condition (1) is satisfied for $e_{3}=\left\{x_{1}, y_{2}, x_{3}\right\}$. On the contrary, reconsider $e_{3}=\left\{x_{1}, x_{2} y_{3}, y_{1} \vee y_{2}\right\}$. If $e_{3}$ is not a hyperedge, then $x_{1} x_{2} y_{3} \leq z$ or $x_{2} y_{3}\left(y_{1} \vee y_{2}\right) \leq z$, that is, $x_{2} y_{3} y_{1} \leq z$. Then $e_{3}^{\prime}=\left\{x_{1}, x_{2}, y_{3}\right\}$ is a hyperedge satisfying the condition (1) or $e_{4}^{\prime}=\left\{x_{2}, y_{3}, x_{1}\right\}$ is a hyperedge satisfying the condition (1). Let $e_{3}=\left\{x_{1}, x_{2} y_{3}, y_{1} \vee y_{2}\right\}$ be a hyperedge. In a similar way, we consider $\left\{y_{1}, x_{2} y_{3}, x_{1} \vee x_{3}\right\}$. If $e_{4}$ is not a hyperedge, then $y_{1} x_{2} y_{3} \leq z$ or $x_{2} y_{3}\left(x_{1} \vee x_{3}\right) \leq$ $z$, that is, $x_{2} y_{3} x_{1} \leq z$. Then $e^{\prime \prime}{ }_{3}=\left\{y_{1}, x_{2}, y_{3}\right\}$ is a hyperedge satisfying the condition (1) or $e^{\prime \prime}{ }_{4}=$ $\left\{x_{2}, y_{3}, x_{1}\right\}$ is a hyperedge satisfying the condition (1). Assume that $e_{4}=\left\{y_{1}, x_{2} y_{3}, x_{1} \vee x_{3}\right\}$ is a hyperedge. Then we have two hyperedges $e_{3}=\left\{x_{1}, x_{2} y_{3}, y_{1} \vee y_{2}\right\}$ and $e_{4}=\left\{y_{1}, x_{2} y_{3}, x_{1} \vee x_{3}\right\}$ with $e_{3}$ and $e_{4}$ satisfying the condition (2).

Case 2.2. Let $\operatorname{deg}_{G}(a)=1$ for only an element $a$ of $G$. Wlog., suppose that $E(G)=$ $\left\{x_{1} y_{1}, x_{1} y_{2}, x_{2} y_{3}\right\}$. We consider $\left\{x_{2}, x_{3} y_{1}, x_{1} \vee y_{3}\right\}$. If $x_{2}=x_{3} y_{1}$, then $x_{1} x_{2} \leq z$, is a contradiction. If $x_{2}=x_{1} \vee y_{3}$, then $x_{2} y_{2} y_{1} \leq z$ and so the condition (1) is satisfied for $e_{3}=\left\{x_{2}, y_{2}, y_{1}\right\}$. If $x_{1} \vee$ $y_{3}=x_{3} y_{1}$, then $x_{3} y_{1} y_{2} y_{1} \leq z$. In the circumstances, if $x_{3}=y_{1} y_{2}$, then $x_{1} x_{3} \leq z$, a contradiction. If $y_{1}=y_{1} y_{2}$, then $y_{1} y_{3} \leq z$, a contradiction. Hence, the condition (1) holds for $e_{3}=\left\{x_{3}, y_{1} y_{2}, y_{1}\right\}$. Let the above conditions not hold. If $e_{3}=\left\{x_{2}, x_{3} y_{1}, x_{1} \vee y_{3}\right\}$ is not a hyperedge, then $x_{2} x_{3} y_{1} \leq z$ or $x_{3} y_{1}\left(x_{1} \vee y_{3}\right) \leq z$, that is, $x_{3} y_{1} y_{3} \leq z$. Then $e^{\prime}{ }_{3}=\left\{x_{2}, x_{3}, y_{1}\right\}$ is a hyperedge satisfying the condition (1) or $e^{\prime}{ }_{4}=\left\{x_{3}, y_{1}, y_{3}\right\}$ is a hyperedge satisfying the condition (1). Suppose that $e_{3}=$ 
$\left\{x_{2}, x_{3} y_{1}, x_{1} \vee y_{3}\right\}$ is a hyperedge. Now, similarly we consider $\left\{y_{2}, x_{3} y_{1}, y_{3}\right\}$. If $e_{4}=\left\{y_{2}, x_{3} y_{1}, y_{3}\right\}$ is not a hyperedge, then $y_{2} x_{3} y_{1} \leq z$ or $x_{3} y_{1} y_{3} \leq z$. Then $e^{\prime \prime}{ }_{3}=\left\{y_{2}, x_{3}, y_{1}\right\}$ is a hyperedge satisfying the condition (1) or $e_{4}^{\prime}=\left\{x_{3}, y_{1}, y_{3}\right\}$ is a hyperedge satisfying the condition (1). Let $\left\{y_{2}, x_{3} y_{1}, x_{1} \vee y_{3}\right\}$ be a hyperedge. Then we obtain two hyperedges $e_{3}=\left\{x_{2}, x_{3} y_{1}, x_{1} \vee y_{3}\right\}$ and $e_{4}=\left\{y_{2}, x_{3} y_{1}, y_{3}\right\}$ with $e_{3}$ and $e_{4}$ satisfying the condition.

Case 2.3. Let $\operatorname{deg}_{G}(a)=\operatorname{deg}_{G}(b)=2$ for $a, b \in V(G)$. Wlog., suppose that $E(G)=$ $\left\{x_{1} y_{1}, x_{1} y_{2}, x_{2} y_{2}\right\}$. Then $\operatorname{deg}_{G}\left(x_{3}\right)=\operatorname{deg}_{G}\left(y_{3}\right)=0$ and so the proof is completed.

Case 2.4. Let $\operatorname{deg}_{G}(a)=3$ for only one element $a$ of $G$. Wlog., suppose that $E(G)=$ $\left\{x_{1} y_{1}, x_{1} y_{2}, x_{1} y_{3}\right\}$. Let $x_{1}^{2} x_{2} \$ z$. Consider $\left\{x_{1} x_{2} \vee y_{1}, x_{1}, x_{3}\right\}$. If $x_{1} x_{2} \vee y_{1}=x_{1}$, then $y_{2} y_{1} \leq z$, a contradiction. If $x_{1} x_{2} \vee y_{1}=x_{3}$, then $x_{3} y_{3} y_{2} \leq z$, a contradiction. Hence $e_{3}=\left\{x_{3}, y_{2}, y_{3}\right\}$ is a hyperedge satisfying the condition (1). In the other case, $e_{3}=\left\{x_{1} x_{2} \vee y_{1}, x_{1}, x_{3}\right\}$ is a hyperedge. In a similar way, we consider $\left\{x_{1} x_{2} \vee y_{1}, y_{2}, y_{3}\right\}$. Then we have a hyperedge $e_{3}$ which satisfies the condition (1) or $e_{4}=\left\{x_{1} x_{2} \vee y_{1}, y_{2}, y_{3}\right\}$ is a hyperedge with $e_{3}$ and $e_{4}$ satisfying the condition (2). Let $x_{1}^{2} x_{2} \leq z$. We consider $\left\{x_{1} \vee y_{1}, x_{1}, x_{2}\right\}$. If $x_{1} \vee y_{1}=x_{2}$, then $x_{2} y_{3} y_{2} \leq z$, a contradiction. Thus $e_{3}=\left\{x_{1} \vee y_{1}, x_{1}, x_{2}\right\}$ is a hyperedge. In a similar way, we consider $\left\{x_{1} \vee y_{1}, y_{2}, y_{3}\right\}$. Then we have a hyperedge $e_{3}$ which satisfies the condition (1) or $e_{4}=\left\{x_{1} x_{2} \vee y_{1}, y_{2}, y_{3}\right\}$ is a hyperedge with $e_{3}$ and $e_{4}$ satisfying the condition (2).

Case 3. Assume that $|E(G)|=4$. Consider four different subcases for this case:

Case 3.1. Let $\operatorname{deg}_{G}(a)=3$ for only one element $a$ of $G$. Wlog., suppose that $E(G)=$ $\left\{x_{1} y_{1}, x_{1} y_{2}, x_{1} y_{3}, x_{2} y_{3}\right\}$. We consider $\left\{x_{3} y_{1}, x_{2}, x_{1} \vee y_{3}\right\}$. If $x_{3} y_{1}=x_{2}$, then $x_{3} y_{3} y_{1} \leq z, \mathrm{a}$ contradiction. Thus $e_{3}=\left\{x_{3}, y_{1}, y_{3}\right\}$ is a hyperedge which holds (1). If $x_{3} y_{1}=x_{1} \vee y_{3}$, then $x_{1}^{2} \leq$ $z$, is a contradiction. If $x_{2}=x_{1} \vee y_{3}$, then $y_{3}^{2} \leq z$, a contradiction. In the other condition, consider again $e_{3}=\left\{x_{3} y_{1}, x_{2}, x_{1} \vee y_{3}\right\}$. If $e_{3}=\left\{x_{3} y_{1}, x_{2}, x_{1} \vee y_{3}\right\}$ is not a hyperedge, then $x_{2} x_{3} y_{1} \leq z$ or $x_{3} y_{1}\left(x_{1} \vee y_{3}\right) \leq z$, that is, $x_{3} y_{1} y_{3} \leq z$. Then $e_{3}^{\prime}=\left\{x_{2}, x_{3}, y_{1}\right\}$ is a hyperedge satisfying the condition (1) or $e_{4}^{\prime}=\left\{x_{3}, y_{1}, y_{3}\right\}$ is a hyperedge satisfying the condition (1). Assume that $e_{3}=$ $\left\{x_{3} y_{1}, x_{2}, x_{1} \vee y_{3}\right\}$ is a hyperedge. In a similar way, we consider $\left\{x_{3} y_{1}, y_{2}, y_{3}\right\}$. If $e_{4}=$ $\left\{x_{3} y_{1}, y_{2}, y_{3}\right\}$ is not a hyperedge, then $x_{3} y_{1} y_{2} \leq z$ or $x_{3} y_{1} y_{3} \leq z$. Then $e^{\prime \prime}{ }_{3}=\left\{y_{2}, x_{3}, y_{1}\right\}$ is a hyperedge satisfying the condition (1) or $e_{4}^{\prime}=\left\{x_{3}, y_{1}, y_{3}\right\}$ is a hyperedge satisfying the condition (1). Suppose that $e_{4}=\left\{x_{3} y_{1}, y_{2}, y_{3}\right\}$ is a hyperedge. Then we get two hyperedges $e_{3}=$ $\left\{x_{3} y_{1}, x_{2}, x_{1} \vee y_{3}\right\}$. and $e_{4}=\left\{x_{3} y_{1}, y_{2}, y_{3}\right\}$ with $e_{3}$ and $e_{4}$ satisfying the condition (2).

Case 3.2. Assume that the degree of four vertices of $G$ equals to two. Wlog., presume that $E(G)=$ $\left\{x_{1} y_{1}, x_{1} y_{2}, x_{2} y_{1}, x_{2} y_{2}\right\}$. Then $\operatorname{deg}_{G}\left(x_{3}\right)=\operatorname{deg}_{G}\left(y_{3}\right)=0$ and so the proof is completed.

Case 3.3. Suppose that the degree of three vertices of $G$ is two. Wlog. assume that $E(G)=$ $\left\{x_{1} y_{1}, x_{1} y_{2}, x_{2} y_{2}, x_{2} y_{3}\right\}$. We consider $\left\{x_{3} y_{3}, x_{1}, x_{2}\right\}$. If $x_{3} y_{3}=x_{1}$ or $x_{3} y_{3}=x_{2}$, then $x_{3} y_{3} y_{2} \leq z$ and so (1) is satisfied for a hyperedge $e_{3}=\left\{x_{3}, y_{2}, y_{3}\right\}$. In the other case, let us view $e_{3}=$ $\left\{x_{3} y_{3}, x_{1}, x_{2}\right\}$. If $e_{3}=\left\{x_{3} y_{3}, x_{1}, x_{2}\right\}$ is not a hyperedge, then $x_{3} y_{3} x_{1} \leq z$ or $x_{3} y_{3} x_{2} \leq z$. Then $e_{3}^{\prime}=$ $\left\{x_{3}, y_{3}, x_{1}\right\}$ is a hyperedge satisfying the condition (1) or $e^{\prime}{ }_{4}=\left\{x_{3}, y_{3}, x_{2}\right\}$ is a hyperedge satisfying the condition (1). Let $e_{3}=\left\{x_{3} y_{3}, x_{1}, x_{2}\right\}$ be a hyperedge. In a similar way, we consider $\left\{x_{3} y_{3}, y_{1}, y_{2}\right\}$. If $e_{4}=\left\{x_{3} y_{3}, y_{1}, y_{2}\right\}$ is not a hyperedge, then $x_{3} y_{3} y_{1} \leq z$ or $x_{3} y_{3} y_{2} \leq z$. Then $e^{\prime \prime}{ }_{3}=\left\{x_{3}, y_{3}, y_{1}\right\}$ is a hyperedge satisfying the condition (1) or $e^{\prime \prime}{ }_{4}=\left\{x_{3}, y_{3}, y_{2}\right\}$ is a hyperedge 
satisfying the condition (1). Let $e_{4}=\left\{x_{3} y_{3}, y_{1}, y_{2}\right\}$ be a hyperedge. Then we get two hyperedges $e_{3}=\left\{x_{3} y_{3}, x_{1}, x_{2}\right\}$ and $e_{4}=\left\{x_{3} y_{3}, y_{1}, y_{2}\right\}$ with $e_{3}$ and $e_{4}$ satisfying the condition (2).

Case 3.4. Let $\operatorname{deg}_{G}(a)=\operatorname{deg}_{G}(b)=2$ for $a, b \in V(G)$. Then, we have two different cases and we can choose one of these sets $E(G)=\left\{x_{1} y_{1}, x_{1} y_{2}, x_{2} y_{2}, x_{3} y_{3}\right\}$ and $E(G)=\left\{x_{1} y_{1}, x_{1} y_{2}, x_{2} y_{3}, x_{3} y_{3}\right\}$. In the first choice, we consider $\left\{x_{3} y_{1}, x_{2}, x_{1} \vee y_{2}\right\}$. If $x_{3} y_{1}=x_{2}$, then $x_{3} y_{1} y_{2} \leq z$ and so $e_{3}=$ $\left\{x_{3}, y_{1}, y_{2}\right\}$ is an edge satisfying (1). If $x_{3} y_{1}=x_{1} \vee y_{2}$, then $x_{1}^{2} \leq z$, a contradiction. If $x_{2}=x_{1} \vee$ $y_{2}$, then $y_{2}^{2} \leq z$, is a contradiction. In the other case, consider $e_{3}=\left\{x_{3} y_{1}, x_{2}, x_{1} \vee y_{2}\right\}$. If $e_{3}=$ $\left\{x_{3} y_{1}, x_{2}, x_{1} \vee y_{2}\right\}$ is not a hyperedge, then $x_{3} y_{1} x_{2} \leq z$ or $x_{3} y_{1}\left(x_{1} \vee y_{2}\right) \leq z$, that is, $x_{3} y_{1} y_{2} \leq z$. Then $e^{\prime \prime}{ }_{3}=\left\{x_{3}, y_{1}, y_{2}\right\}$ is a hyperedge satisfying the condition (1) or $e_{4}^{\prime \prime}=\left\{x_{3}, y_{1}, y_{3}\right\}$ is a hyperedge satisfying the condition (1). Let $e_{4}=\left\{x_{3} y_{1}, y_{2}, y_{3}\right\}$ be a hyperedge. Then we get two hyperedges $e_{3}=\left\{x_{3} y_{1}, x_{2}, x_{1} \vee y_{2}\right\}$ and $e_{4}=\left\{x_{3} y_{1}, y_{2}, y_{3}\right\}$ with $e_{3}$ and $e_{4}$ satisfying the condition (2).

In a similar manner, we consider $\left\{x_{1} \vee y_{1}, x_{2}, x_{3}\right\}$ and $\left\{x_{1} \vee y_{1}, y_{2}, y_{3}\right\}$ for the second choice. Hence, we have a hyperedge $e_{3}$ which holds (1) or two hyperedges $e_{3}$ and $e_{4}$ which hold the condition (2).

Case 4. Assume that $|E(G)|=5$. Consider four different subcases for this case:

Case 4.1. Wlog. assume that $E(G)=\left\{x_{1} y_{1}, x_{1} y_{2}, x_{1} y_{3}, x_{2} y_{1}, x_{2} y_{2}\right\}$. We consider $\left\{x_{3} y_{3}, x_{2}, x_{1} \vee\right.$ $\left.y_{2}\right\}$. If $x_{3} y_{3}=x_{2}$, then $x_{3} y_{3} x_{2} \leq z$, and so the condition (1) is satisfied for a hyperedge $e_{3}=$ $\left\{x_{2}, x_{3}, y_{3}\right\}$. If $x_{3} y_{3}=x_{1} \vee y_{2}$, then $x_{1}^{2} \leq z$, a contradiction. If $x_{2}=x_{1} \vee y_{2}$, then $y_{1} y_{2} \leq z$, yielding a contradiction. On the other hand, $e_{3}=\left\{x_{3} y_{3}, x_{2}, x_{1} \vee y_{2}\right\}$ is a edge in $G$. In a similar way, we consider $\left\{x_{3} y_{3}, y_{1}, y_{2}\right\}$. If $e_{4}=\left\{x_{3} y_{3}, y_{1}, y_{2}\right\}$ is not a hyperedge, then $x_{3} y_{3} y_{1} \leq z$ or $x_{3} y_{3} y_{2} \leq$ $z$. Then $e^{\prime \prime}{ }_{3}=\left\{x_{3}, y_{3}, y_{1}\right\}$ is a hyperedge satisfying the condition (1) or $e^{\prime \prime}{ }_{4}=\left\{x_{3}, y_{3}, y_{2}\right\}$ is a hyperedge satisfying the condition (1). Let $e_{4}=\left\{x_{3} y_{3}, y_{1}, y_{2}\right\}$ be a hyperedge. Then we get two hyperedges $e_{3}=\left\{x_{3} y_{3}, x_{2}, x_{1} \vee y_{2}\right\}$ and $e_{4}=\left\{x_{3} y_{3}, y_{1}, y_{2}\right\}$ with $e_{3}$ and $e_{4}$ satisfying the condition (2).

Case 4.2. Wlog., presume that $E(G)=\left\{x_{1} y_{1}, x_{1} y_{2}, x_{1} y_{3}, x_{2} y_{1}, x_{3} y_{2}\right\}$. We consider $\left\{x_{1} \vee\right.$ $\left.y_{1}, x_{2}, y_{2}\right\}$. If $x_{1} \vee y_{1}=x_{2}$, then $y_{1}^{2} \leq z$, is a contradiction. If $x_{1} \vee y_{1}=y_{2}$, then $x_{1}^{2} \leq z$, is a contradiction. In the following situations, $e_{3}=\left\{x_{1} \vee y_{1}, x_{2}, x_{3} y_{3}\right\}$ is a hyperedge of $G$ satisfying (1).

Case 4.3. Wlog., presume that $E(G)=\left\{x_{1} y_{1}, x_{1} y_{2}, x_{1} y_{3}, x_{2} y_{1}, x_{3} y_{2}\right\}$. We consider $\left\{x_{1} \vee\right.$ $\left.y_{1}, x_{2}, y_{2}\right\}$. If $x_{1} \vee y_{1}=x_{2}$ then $y_{1}^{2} \leq z$, is a contradiction. If $x_{1} \vee y_{1}=y_{2}$ then $x_{2} x_{3} y_{2} \leq z$. Thus $e_{3}=\left\{x_{2}, x_{3}, y_{2}\right\}$ is a hyperedge satisfying (1). In the other case, $e_{3}=\left\{x_{1} \vee y_{1}, x_{2}, y_{2}\right\}$ is a hyperedge satisfying (1).

Case 4.4. Wlog., let $E(G)=\left\{x_{1} y_{1}, x_{1} y_{2}, x_{2} y_{1}, x_{2} y_{2}, x_{3} y_{3}\right\}$. We consider $\left\{x_{3} \vee y_{1}, x_{1}, y_{3}\right\}$. If $x_{3} \vee$ $y_{1}=x_{1}$ or $x_{3} \vee y_{1}=y_{3}$, then $x_{1} x_{2} y_{3} \leq z$. Then $e_{3}=\left\{x_{1}, x_{2}, y_{3}\right\}$ is a hyperedge satisfying the condition (1). In the other case, $e_{3}=\left\{x_{3} \vee y_{1}, x_{1}, y_{3}\right\}$ is a hyperedge satisfying the condition (1).

Case 4.5. Wlog., presume that $E(G)=\left\{x_{1} y_{1}, x_{1} y_{2}, x_{2} y_{2}, x_{2} y_{3}, x_{3} y_{3}\right\}$. We consider $\left\{x_{1} \vee\right.$ $\left.y_{2}, x_{2}, y_{1}\right\}$. If $x_{1} \vee y_{2}=x_{2}$, then $y_{2}^{2} \leq z$, is a contradiction. If $x_{1} \vee y_{2}=y_{1}$, then $x_{1}{ }^{2} \leq z$, is a contradiction. Then $e_{3}=\left\{x_{1} \vee y_{2}, x_{2}, y_{1}\right\}$ is a hyperedge satisfying the condition (1). 
Case 5. Let $|E(G)|=6$. Consider three different subcases for this case:

Case 5.1. Wlog., presume that $E(G)=\left\{x_{1} y_{1}, x_{1} y_{2}, x_{1} y_{3}, x_{2} y_{1}, x_{2} y_{2}, x_{3} y_{1}\right\}$.

We consider $\left\{x_{1} \vee y_{1}, x_{2}, x_{3}\right\}$ and $\left\{x_{1} \vee y_{1}, y_{2}, y_{3}\right\}$. If $x_{1} \vee y_{1}=x_{2}$, then $y_{1} y_{2} \leq z$, a contradiction. If $x_{1} \vee y_{1}=x_{3}$, then $y_{1}{ }^{2} \leq z$, is a contradiction. If $x_{1} \vee y_{1}=y_{2}$ or $x_{1} \vee y_{1}=y_{3}$, then $x_{1}{ }^{2} \leq z$, is a contradiction. Thus $e_{3}=\left\{x_{1} \vee y_{1}, x_{2}, x_{3}\right\}$ and $e_{4}=\left\{x_{1} \vee y_{1}, y_{2}, y_{3}\right\}$ are hyperedges satisfying the condition (2).

Case 5.2. Wlog., presume that $E(G)=\left\{x_{1} y_{1}, x_{1} y_{2}, x_{1} y_{3}, x_{2} y_{1}, x_{2} y_{2}, x_{3} y_{3}\right\}$.

We consider $\left\{x_{1} \vee y_{3}, x_{3}, y_{1}\right\}$. If $x_{1} \vee y_{3}=x_{3}$, then $y_{3}{ }^{2} \leq z$, is a contradiction. If $x_{1} \vee y_{3}=y_{1}$, then $x_{1}{ }^{2} \leq z$, is a contradiction. Thus $e_{3}=\left\{x_{1} \vee y_{3}, x_{3}, y_{1}\right\}$ is a hyperedge satisfying the condition (1).

Case 5.3. Wlog., presume that $E(G)=\left\{x_{1} y_{1}, x_{1} y_{3}, x_{2} y_{1}, x_{2} y_{2}, x_{3} y_{2}, x_{3} y_{3}\right\}$. We consider $\left\{x_{1} \vee\right.$ $\left.y_{3}, x_{3}, y_{1}\right\}$. If $x_{1} \vee y_{3}=x_{3}$, then $y_{3}^{2} \leq z$, is a contradiction. If $x_{1} \vee y_{3}=y_{1}$, then $x_{1}{ }^{2} \leq z$, is a contradiction. Thus $e_{3}=\left\{x_{1} \vee y_{3}, x_{3}, y_{1}\right\}$ is a hyperedge satisfying the condition (1).

Case 6. If $7 \leq|E(G)| \leq 9$, then we have two vertices which are degree three in $e_{1}$ and the other in $e_{2}$. We suppose that $\operatorname{deg}_{G}\left(x_{1}\right)=\operatorname{deg}_{G}\left(y_{1}\right)=3$. We consider $\left\{x_{1} \vee y_{1}, x_{2}, x_{3}\right\}$ and $\left\{x_{1} \vee y_{1}, y_{2}, y_{3}\right\}$. If $x_{1} \vee y_{1}=x_{2}$ or $x_{1} \vee y_{1}=x_{3}$, then $y_{1}^{2} \leq z$, is a contradiction. If $x_{1} \vee y_{1}=y_{2}$ or $x_{1} \vee y_{1}=y_{3}$, then $x_{1}{ }^{2} \leq z$, is a contradiction. Hence $e_{3}=\left\{x_{1} \vee y_{1}, x_{2}, x_{3}\right\}$ and $e_{4}=\left\{x_{1} \vee y_{1}, y_{2}, y_{3}\right\}$ are hyperedges satisfying the condition (2).

By the fact that $\operatorname{gr}\left(H_{3}(L, z)\right) \leq 2 \operatorname{diam}\left(H_{3}(L, z)\right)+1$, we have that $\operatorname{gr}\left(H_{3}(L, z)\right) \leq 9$.

\subsection{Complete 3-Partite Hypergraph}

Definition 2. [10] A hypergraph $H$ is called an $n$-partite if the vertex set $V$ can be partitioned into disjoint subsets $V_{1}, V_{2}, \ldots, V_{n}$ of $V$ such that a hyperedge in the hyperedge set $E$ composes of a choice of completely one vertex from each subset of $V$. Also, a hypergraph $H$ is called a complete $n$-partite hypergraph if the vertex set $V$ can be partitioned into disjoint subsets $V_{1}, V_{2}, \ldots, V_{n}$ of $V$ and each element of $V_{i}$ for each $1 \leq i \leq n$ creates a hyperedge of $H$.

Proposition 2. Let $H_{3}(L, z)$ be a complete 3-partite hypergraph.

If $x y \leq z$, then $x$ and $y$ are contained by same subset $V_{i}$ for some $i \in\{1,2,3\}$.

Proof. Let $H_{3}(L, z)$ has disjoint subsets $V_{1}, V_{2}, V_{3}$ which are partitions of the vertex set $V$. Let $a$ be a vertex with $x y a \leq z$. Without loss of generality, assume that $x \in V_{1}$ and $a \in V_{2}$. Then $e=\{x, y, a\}$ is not a hyperedge in $H_{3}(L, z)$ by our assumption. If $y \in V_{3}$, then $e$ is a hyperedge since $H_{3}(L, z)$ is a complete 3-partite hypergraph, a contradiction. If $y \in V_{2}$, then there is a vertex $b \in V_{3}$ such that $e^{\prime}=\{x, y, b\}$. But this contradicts the fact that $x y \leq z$. Therefore, $y$ must be in $V_{1}$.

Theorem 2. Let $z$ be a proper element of $L$. Then the following statements hold:

(1) If $p_{1}, p_{2}$ and $p_{3}$ are prime elements of $L$ and $z=p_{1} \wedge p_{2} \wedge p_{3} \neq 0_{L}$, then $H_{3}(L, z)$ is a complete 3-partite hypergraph. 
(2) Let $a^{2} \leq z$ for every 3-zero-divisor $a \in L$ with respect to $z$ and $H_{3}(L, z)$ be a complete 3partite hypergraph over the reduced lattice $L$. Then there exist prime elements $p_{1}, p_{2}$ and $p_{3}$ of $L$ such that $p_{1} \wedge p_{2} \wedge p_{3} \leq z$.

Proof. (1). Let $e=\{a, b, c\}$ be a hyperedge of $H_{3}(L, z)$. Then $a b c \leq z=p_{1} \wedge p_{2} \wedge p_{3}$, that is, $a b c \leq p_{1}, p_{2}, p_{3}$. Since $p_{i}$ is a prime element for any $i \in\{1,2,3\}$, then $a \leq p_{1}$ or $b \leq p_{1}$ or $c \leq$ $p_{1}$ and $a \leq p_{2}$ or $b \leq p_{2}$ or $c \leq p_{2}$ and $a \leq p_{3}$ or $b \leq p_{3}$ or $c \leq p_{3}$. Additionally, $a b \$ p_{i}$ and $b c \$ p_{j}$ and $a c \$ p_{k}$ for some $i, j, k \in\{1,2,3\}$ since $a b, b c, a c \$ z=p_{1} \wedge p_{2} \wedge p_{3}$. Wlog., we assume $a b \$ p_{1}$. Then $a \$ p_{1}$ and $b \$ p_{1}$. Thus, we have $c \leq p_{1}$. Indeed, if $a c \$ p_{1}$, then $b \leq$ $p_{1}$, a contradiction. In a similar manner, suppose that $a c \$ p_{2}$. Then $a \$ p_{2}$ and $c \$ p_{2}$. Thus, this yields $b \leq p_{2}$. Indeed, if $b c \$ p_{1}$, then $a \leq p_{1}$, a contradiction and if $b c \$ p_{2}$, then $a \leq p_{2}$, a contradiction. Thus, it must be $b c \$ p_{3}$. Then, we get $a \leq p_{3}$. We assume that $a \leq p_{3}$ and $a \ddagger$ $p_{1}, p_{2}, b \leq p_{2}$ and $b \$ p_{1}, p_{3}$ and $c \leq p_{1}$ and $c \$ p_{2}, p_{3}$. Consequently, $H_{3}(L, z)$ is a complete 3-partite hypergraph with parts $V_{i}$ for any $i \in\{1,2,3\}$ whose vertices must be only less than or equal to $p_{i}$.

(2). Let $H_{3}(L, z)$ be a complete 3-partite hypergraph and it has parts $V_{1}, V_{2}$ and $V_{3}$. Set $p_{1}=V_{1} \vee$ $z, p_{2}=V_{2} \vee z$ and $p_{3}=V_{3} \vee z$. Then $x_{1} x_{2} x_{3} \leq z$ for every $x_{i} \leq p_{i}$ for any $i \in\{1,2,3\}$. It is clear that $\left(\mathrm{V}_{x_{1} \in V_{1}} x_{1}\right)\left(\mathrm{V}_{x_{2} \in V_{2}} x_{2}\right)\left(\mathrm{V}_{x_{3} \in V_{3}} x_{3}\right) \vee z \leq z$, that is, $p_{1} p_{2} p_{3} \leq z$ since $L$ is a multiplicative lattice. As $L$ is reduced, then $p_{1} \wedge p_{2} \wedge p_{3} \leq z$. We assume that $p_{1}$ is not a prime element of $L$, that is, $a b \leq p_{1}$ and $a, b \leq p_{1}$ for some $a, b \in L$. Since $a b \leq p_{1}=V_{1} \vee z$ then $a b \leq z$ or $a b \in$ $V_{1}$. We have three cases for this assumption.

Case 1. Let $a b \in V_{1}$ and $a b \leq z$. This contradicts the definition of vertex set of $H_{3}(L, z)$.

Case 2. Let $a b \in V_{1}$ and $a b \$ z$. Since $a b \in V_{1}$ and $a \notin V_{1}$, then $a \in V_{2}$ or $a \in V_{3}$. Wlog., assume that $a \in V_{2}$. So, $\{a b, a, c\}$ must be a hyperedge of $H_{3}(L, z)$ for any $c \in V_{3}$. However, since $a^{2} \leq z$ for every 3-zero-divisor $a \in L$, then $a^{2} b \leq z$, contradiction.

Case 3. Let $a b \notin V_{1}$ and $a b \leq z$. By Proposition 2, $a$ and $b$ must be in the same $V_{i}$ for any $i=$ $\{2,3\}$. Wlog., let $a, b \in V_{2}$. Then, $x a y \leq z, x a \$ z, x y \$ z, a y \ddagger z$ and $x b y \leq z, x b \$ z, x y \ddagger$ $z$, by $₫ z$ for some $x \in V_{1}$ and $y \in V_{3}$. By Proposition 2, we obtain that $x a \in V_{3}, x b \in V_{3}$, ay $\in$ $V_{1}$, by $\in V_{1}$. Therefore, $\{a y, b, x a\}$ must be a hyperedge, since $H_{3}(L, z)$ is a complete 3-partite hypergraph. However, $a^{2} y x \leq z$ for $a^{2} \leq z$, contradiction. We have a contradiction for each cases. Therefore, $a$ or $b$ must be less than or equal to $p_{1}$. Similarly, it can be seen that $p_{2}$ and $p_{3}$ are prime elements in $L$.

\subsection{Cut Points and Bridge of $H_{3}(L, z)$}

Definition 3. [6] A vertex $a$ of a connected graph $G$ is called a cut-point of $G$ if there are vertices $x$ and $y$ of $G$ with $a \neq x$ and $a \neq y$ such that $a$ is in every path which is from $x$ to $y$.

Theorem 3. Let $z \in L$ and $S=\{u \in L \mid u \leq z$ and $u \nsubseteq a\}$. If $S \neq \emptyset$, then $a$ is not a cut-point in $H_{3}(L, z)$. 
Proof. Let $a$ be in every path which is from $x$ to $y$ with $a \neq x$ and $a \neq y$. We know that $d(x, y)=$ 2, 3 or 4 by Theorem 1 . Consider $a \vee u$. Note that it is a vertex in $H_{3}(L, z)$ which is different from $a$. We consider the following cases:

Case 1. Let $d(x, y)=2$. Then there are two hyperedges $e_{1}=\left\{x, a, c_{1}\right\}$ and $e_{2}=\left\{a, y, c_{2}\right\}$ for some vertices $c_{1}, c_{2}$ in $H_{3}(L, z)$ such that $x-{ }_{e_{1}} a-{ }_{e_{2}} y$ is a path. Consider $e^{\prime}{ }_{1}=\left\{x, a \vee u, c_{1}\right\}$ and $e_{2}^{\prime}=\left\{a \vee u, y, c_{2}\right\}$

Let $a \vee u \neq x, a \vee u \neq y$ and $a \vee u \neq c_{i}$ for $i \in\{1,2\}$. It is easily seen that $e^{\prime}{ }_{1}$ and $e^{\prime}{ }_{2}$ are two hyperedges such that $x-{ }_{e_{1}} a \vee u-{ }_{e_{2}} y$ is a path.

i.If $a \vee u=x$ or $a \vee u=y$, then $x$ and $y$ are adjacent.

ii.Consider $a \vee u=c_{1}$ or $a \vee u=c_{2}$. Wlog., assume that $a \vee u=c_{1}$. Then $e^{\prime \prime}{ }_{1}=\{x, a \vee u, a\}$ and $e_{2}^{\prime}=\left\{a \vee u, y, c_{2}\right\}$ are two hyperedges such that $x-e_{\prime^{\prime \prime}} a \vee u-{ }_{e_{2}} y$ is a path.

Thus $a$ is not a cut point.

Case 2. Let $d(x, y)=3$. Then there are three hyperedges $e_{1}=\left\{x, a, c_{1}\right\}$ and $e_{2}=\left\{a, b, c_{2}\right\}$ and $e_{3}=\left\{b, y, c_{3}\right\}$ for some vertices $b, c_{1}, c_{2}, c_{3}$ in $H_{3}(L, z)$ such that $x-e_{1} a-e_{2} b-e_{3} y$ is a path. If $a \vee u$ is different from each of $x, b$ and $c_{i}$ for $i \in\{1,2,3\}$, then there is a path from $x$ to $y$ which does not contain $a$. Now, we consider other situations.

i. Let $a \vee u=x$. Then consider $e_{2}^{\prime}=\left\{a \vee u, b, c_{2}\right\}$ and $e_{3}$. Note that there is a path $a \vee$ $u-e_{e_{2}} b-e_{3} y$. Thus $a$ is not a cut point.

ii. Let $a \vee u=b$. Consider $e_{1}^{\prime}=\left\{x, a \vee u, c_{1}\right\}$ and $e_{3}$. Clearly, there is a path $x-e_{e_{1}} a \vee$ $u-e_{3} y$. Hence $a$ is not a cut point.

iii. Let $a \vee u=y$. Consider $e_{1}^{\prime}=\left\{x, a \vee u, c_{1}\right\}$. Thus $x$ and $y$ are adjacent. Hence $a$ is not a cut point.

iv. $a \vee u=c_{i}$ for $i \in\{1,2\}$. It can be seen in a similar way in Case 1 (ii).

v. Let $a \vee u=c_{3}$. Consider $e^{\prime}{ }_{3}=\{b, y, a \vee u\}$ and $e_{1}^{\prime}=\left\{x, a \vee u, c_{1}\right\}$. Then there is a path such that $x-{ }_{e_{1}{ }_{1}} a-{ }_{e^{\prime}} y$.

Case 3. Let $d(x, y)=4$. Then there are four hyperedges $e_{1}=\left\{x, a, c_{1}\right\}$ and $e_{2}=\left\{a, b, c_{2}\right\}$, $e_{3}=\left\{b, c, c_{3}\right\}$ and $e_{4}=\left\{c, y, c_{4}\right\}$ for some vertices $b, c, c_{1}, c_{2}, c_{3}, c_{4}$ in $H_{3}(L, z)$ such that $x-e_{1} a-e_{2} b-e_{3} y-e_{4} c$ is a path. If $a \vee u$ is different from each of $x, b, c, y$ and $c_{i}$ for $i \in$ $\{1,2,3,4\}$, then there is a path from $x$ to $y$ which does not contain $a$. Now, we consider other situations.

i. Let $a \vee u=x$. Now, consider $e^{\prime}{ }_{2}=\left\{a \vee u, b, c_{2}\right\}$. Then note that $e_{2}^{\prime}$ is a hyperedge and there is a path $a \vee u-{ }_{e_{2}} b-e_{3} c-e_{4} y$.

ii. Let $a \vee u=b$ Consider $e_{1}^{\prime}=\left\{x, a \vee u, c_{1}\right\}$ and $e^{\prime}{ }_{3}=\left\{a \vee u, c, c_{3}\right\}$. Then note that $e^{\prime}{ }_{1}$ and $e^{\prime}{ }_{3}$ are two hyperedges and there is a path $x-{ }_{e_{1} 1_{1}} a \vee u-{ }_{e_{3}} c-e_{4} y$.

iii. Let $a \vee u=c$. Consider $e_{1}^{\prime}=\left\{x, a \vee u, c_{1}\right\}$ and $e_{4}^{\prime}=\left\{a \vee u, y, c_{4}\right\}$. Then note that $e_{4}^{\prime}$ is a hyperedge and there is a path $\mathrm{x}-{ }_{e^{\prime}{ }_{1}} a \vee u-{ }_{e^{\prime}} y$.

iv. Let $a \vee u=y$. Consider $e^{\prime}{ }_{1}=\left\{x, a \vee u, c_{1}\right\}$. Note that $e^{\prime}{ }_{1}$ is a hyperedge and $x$ and $y$ are adjacent.

iv. Let $a \vee u=c_{i}$ for $i \in\{1,2\}$. It can be seen in a similar way in Case 1 (ii). 
v. Let $a \vee u=c_{i}$ for $i \in\{3,4\}$. It can be seen in a similar way in Case $2(\mathrm{v})$.

We obtain the following result by the previous theorem.

Corollary 1. Let $a$ be a vertex in $H_{3}(L, z)$ and $z \Varangle a$. Then $a$ is not a cut-point of $H_{3}(L, z)$.

Proposition 3. If $H_{3}(L, Z)$ is connected, then $H_{3}(L, Z)$ has not any bridge.

Proof. Let $e=\{a, b, c\}$ be a bridge of $H_{3}(L, z)$. Then $H_{3}(L, z)$ is disconnected if $e$ is omitted in hypergraph. Take an element $y$ with $0_{L} \neq y \leq z$. Then $a \vee y, b \vee y, c \vee y \leq z$. Also each of $e_{1}=$ $\{a \vee y, b, c\}, e_{2}=\{a, b \vee y, c\}$ and $e_{3}=\{a, b, c \vee y\}$ is a hyperedge. Thus, there is a cycle $a-e_{3} b-e_{1} c-e_{2} a$. Indeed if $e$ is omitted in hypergraph, $H_{3}(L, z)$ is connected. Thus, $H_{3}(L, z)$ has not any bridge.

\section{REFERENCES}

[1] Jayaram C. and Johnson E.W., Some Results on Almost Principal Element Lattices, Period. Math. Hungar, 31 (1995) 33-42.

[2] Anderson D.D., Abstract Commutative Ideal Theory without Chain Condition, Algebra Universalis, 6 (1976) 131-145.

[3] Anderson D.F. and Livingston P.S., The Zero Divisor of a Commutative Ring, J. of Algebra, (1999) 434-447.

[4] Dilworth R.P., Abstract Commutative Ideal Theory, Pacific Journal of Mathematics 12 (1962) 481-498.

[5] Eslahchi Ch. and Rahimi A.M., The k-Zero-Divisor Hypergraph of a Commutative Ring, Int. J. Math. Math. Sci. Art. 50875 (2007) 15.

[6] Beck I., Coloring of Commutative Rings, J. of Algebra, (1988) 208-226.

[7] Selvakumar K. and Ramanathana V., Classification of non-Local Rings with Genus One 3zero-divisor Hypergraphs, Comm. Algebra, (2016) 275-284.

[8] Akbari S. and Mohammadian A., On the Zero-Divisor Graph of a Commutative Ring, J. Algebra, (2004) 847-855.

[9] Elele A.B. and Ulucak G., 3-Zero-Divisor Hypergraph Regarding an Ideal, 7 th International Conference on Modeling, Simulation, and Applied Optimization (ICMSAO), 2017.

[10] Badawi A., On 2-absorbing Ideals of Commutative Rings, Bull. Austral. Math. Soc.,75 (2007) 417-429. 